\title{
Comment on Fitzgerald and Kirkham: "Average uncertainty" as a variable in backward visual masking
}

HELEN W. STREICHER

INSTITUTE FOR JUVENILE RESEARCH, CHICAGO

It is suggested that the term "average uncertainty" is inappropriate for the description of random checkerboard patterns. Backward visual masking data reported by Fitzgerald $\mathcal{E}$ Kirkham (1966) are discussed as being the result of confounding two contour variables, parallelness and proximity to test figure lines, in the masking stimuli.

Fitzgerald \& Kirkham (1966) studied backward visual masking of horizontal and vertical lines by random checkerboard patterns on $50 \times 50$ matrices. They assumed that masking would be greatest when the amount of information contained in the masking stimulus was greatest, or when the "average uncertainty," based on the probabilities of black and white elements in the matrix, was maximum (probabilities of black and white equal). The stimuli which they used were generated by computer programs in which the probability of blacking in a given element was $0.0,0.2,0.4,0.6,0.8$, or 1.0 . They found that the 0.4 and 0.6 stimuli produced most masking and that the masking function closely approached a plot of average uncertainty.

There would seem to be several difficulties with this approach. In the first place, it is inappropriate to speak of an already-generated stimulus as having a probability of a given magnitude. Once the stimulus has been produced, each element is either black or not, and no statement regarding its probability of being blacked applies. One speaks then in terms of the number or percentage of blacked-in elements. Since it is inappropriate to describe these stimuli in terms of the probability of blacked-in elements, it is also inappropriate to designate their average uncertainty.

Second, even if the concept of information could be meaningfully applied here, there is evidence contradicting the supposition that increasing the amount of information in the masking stimulus will increase the amount of masking. For example, a hexagona' ring, which contains angles, would seem logically to contain more information than a circular ring, since more questions would be required in order to determine that an unidentified line figure was a hexagon, i.e., more "bits." However, a disk figure is masked less by a hexagonal ring than by a circular ring. Further, a hexagonal test figure is masked more by a hexagonal ring than is a disk test figure (Pollack, 1965).
If we accept the view that the concept of average uncertainty does not pertain directly to Fitzgerald \& Kirkham's data, then how are we to account for the close fit of their masking data to the theoretical expectation based on average uncertainty? For the answer to this, it would be useful to ask what their masking stimuli might have looked like. That is, we might examine the physical properties of the masking stimuli and their relationship to those of the stimuli to be masked. Given a $50 \times 50$ matrix and a program in which the probability of blacking in a given element is, for example, 0.5 , the finished product will generally turn out to be about half black, half white. It is highly improbable that the whole top half of the resulting stimulus would be black and the bottom half white. It is also improbable that the result would be a perfect checkerboard with every other element blacked in. What is more usually the case is that some of the blacked-in elements will appear as rows or blocks, forming contours with similar rows of white elements. Since the total matrix is square and the elements are square, all contours thus formed are either vertical or horizontal. In a 0.2 probability program, there will be few of these relatively long contours, and the same would be true of a 0.8 program, which would generate a nearly all-black stimulus. Little or no masking would be expected with an all-white or all-black stimulus, since there would be no contours parallel to the test figure lines which were close enough to those lines to interact with them (Streicher \& Pollack, 1967). If the masking stimulus were $40 \%$ or $60 \%$ black, however, the pattern would probably provide several blackwhite contours close to the area in which the lines had been presented. Further, as has been noted, all of these contours would be either vertical or horizontal, and therefore some would be parallel to the vertical line test figure and some parallel to the horizontal line test figure.

The presence of these horizontal and vertical contours, and their location, is fundamental to the description of the masking stimuli, since contour orientation, intercontour distance (proximity), and contrast are the only variables which have been shown to affect the amount of masking (with the exception of procedural variables such as test figure duration or interstimulus interval). Contour overlap has also been mentioned (Schiller \& Wiener, 1963; 
Schiller, 1966), but this variable, as it has been studied, may be only a combination of the variables of contour orientation and proximity, with complete overlap (identical stimuli superimposed) being the limiting case of both.

In order to test the hypothesis that it is parallelness and proximity of contours in the masking figure to the lines presented as test figure that account for the large amount of masking obtained with the 0.4 and 0.6 matrices, the test figures could be rotated $45^{\circ}$, so that the test figure lines would not be parallel to contours in the masking stimuli. Unless some stimulus characteristic other than proximity and orientation of contours were responsible for the obtained differences in masking, this procedure should depress the masking function.

\section{References}

Fitzgerald, R. E., \& Kirkham, R. Backward visual masking as a function of average uncertainty of the masking pattern. Psychon. Sci., 1966, 6, 469-470.

Pollack, R. H. Effects of figure-ground contrast and contour orientation on figural masking. Psychon. Sci., 1965, 2, 369-370.

Schiller, P. H. Forward and backward masking as a function of relative overlap and intensity of test and masking stimuli. Percept. \& Psychophys., 1966, 1, 161-164.

Schiller, P. H., \& Wiener, M. Monoptic and dichoptic masking. $J$. exp. Psychol., 1963, 66, 386-393.

Streicher, Helen W., \& Pollack, R. H. Backward figural masking as a function of intercontour distance. Psychon. Sci., 1967, 7, 69-70. 